\title{
The impact of spatiotemporal sampling on time-to-contact judgments
}

\author{
HEIKO HECHT \\ MIT Man-Vehicle Lab, Cambridge, Massachusetts \\ MARY K. KAISER \\ NASA Ames Research Center, Moffet Field, California \\ and \\ GEERT J. P. SAVELSBERGH and JOHN VAN DER KAMP \\ Vrije Universiteit, Amsterdam, The Netherlands
}

\begin{abstract}
When motion in the frontoparallel plane is temporally sampled, it is often perceived to be slower than its continuous counterpart. This finding stands in contrast to humans' ability to extrapolate and anticipate constant-velocity motion. We investigated whether this sampling bias generalizesto motion in the sagittal plane (i.e., objects approaching the observer). We employed a paradigm in which observers judged the arrival time of an oncoming object. We found detrimental effects of time sampling on both perceived time to contact and time to passage. Observers systematically overestimated the time it would take a frontally approaching object to intersect their eye plane. To rule out artifactsinherent in computer simulation, we replicated the experiment, using real objects. The bias persisted and proved to be robust across a large range of temporal and spatial variations. Energy and pooling mechanisms are discussed in an attempt to understand the effect.
\end{abstract}

Observers are generally accurate when judging temporal aspects of objects moving laterally in front of them, such as velocity or arrival time (e.g., Gottsdanker, 1952; Schiff $\&$ Oldak, 1990). However, when the stimulus is sampled so that it flashes on and off during its motion, errors are made (e.g., Hecht \& Hoffman, 2000; Hecht, Oesker, Kaiser, Civelek, \& Stecker, 1999). For instance, in a standard motion extrapolation task, an object traverses the screen and then disappears. Observers have to judge when it would have reached a designated spot farther along on its trajectory. If and only if the visible portion of the trajectory is intermittent do observers judge arrival times to be later, as if the sampled object moved slower than its continuously visible counterpart. We call this the sampling effect.

A large body of literature that dates back to the early days of Gestalt psychology indirectly speaks to this phenomenon. It can be summarized under the heading of spatiotemporal dissociation and will be briefly described in the fol-

Portions of this research were presented at the 37th Annual Meeting of the Psychonomic Society. H.H.'s work was supported by the Deutsche Forschungsgemeinschaft (DFG Equipment Grant He 2122/4-1+2); J.v.d.K. was supported by the Foundation for Behavioral Sciences (Grant 575-59-055), which is funded by the Netherlands Organization for Scientific Research (NWO). The authors thank Don Hoffman and James Todd for stimulating discussions and Felix Shung for his excellent programming support. John Andersen and two anonymous reviewers provided valuable comments on an earlier version of this paper. Correspondence concerning this article should be addressed to H. Hecht, MIT Man-Vehicle Lab, 77 Massachusetts Ave., Bldg. 37-219, Cambridge, MA 02139 (e-mail: hecht@ space.mit.edu). lowing paragraphs. Interestingly, to this date, a satisfactory explanation of such dissociation is lacking. To pave the ground for a better understanding of the sampling effect, we first examined whether it generalizes to motion in the sagittal plane, which it does. We then explored different varieties of sampling and tested whether the effect is confined to computer-generated displays or generalizes to a real-world task.

\section{Sampling of Motion in the Frontoparallel Plane}

The effects of motion sampling and of intermittent viewing have been studied with respect to objects moving in the frontoparallel plane. In most real-life situations, however, objects' motions are not confined to this plane. Classical apparent motion (AM) studies were typically interested in the phenomenal aspects of sampled motion. Temporally and spatially alternating objects were first created on turntables (Wertheimer, 1912) by moving a radial line behind a stationary template that contained different apertures to produce the desired sampling rates. With the introduction of light-emitting diodes (LEDs) and computer displays, discrete sampling became predominant. Aperture sampling has been referred to, somewhat confusingly, as spatial sampling (Dannemiller, Heidenreich, \& Babler, 1997). Obviously, temporal and spatial sampling co-occur so long as the object in question is moving. Thus, we use the term temporal sampling to indicate that the complete object disappeared intermittently and ignore differences between discrete and aperture sampling for the purposes of this study. Also, we are not concerned with perceptually 
smooth phi motion, but rather with extending sampling ranges beyond short-range and even beyond long-range AM (Braddick, 1974).

Spatiotemporal dissociations have been investigated at length by Helson and colleagues, who noticed that our perception of time and motion is often inaccurate because of a perceptual interaction of distance, time, and motion (i.e., pooling mechanisms; see Helson, 1925; Helson \& King, 1931). They discovered that if three points, which are evenly spaced on the skin, are stimulated in uneven time intervals, the points' locations on the skin are misperceived. Locations stimulated at larger time intervals feel farther away than they are, and locations stimulated at short time intervals feel closer together than they are. This so-called tau effect (not to be confused with the use of tau to indicate time to contact [TTC]) has been replicated numerous times for cutaneous, visual, and auditory stimuli (for overviews, see Geldard, 1975; Ono, 1976). The influence of timing on perceived location also has its counterpart, the kappa effect: Brief flashes of light presented at equal time intervals but at unevenly separated locations (e.g., a large separation followed by a small one) produce subjective time distortions (the second time interval is perceived as shorter than the first).

Unfortunately, Helson's explanation for the kappa and tau effects cannot explain the sampling effect once illusory object displacement is ruled out (see Experiment 1). Helson assumes a spatial pooling mechanism that causes a misjudgment of target position (Helson \& Bevan, 1967). That is, the position assigned to the moving target when last visible does not correspond to the position of the final flash but, rather, to the location between the final two flashes. We ruled out such misjudgment in the present data.

A single flash analogue to the sampling effect, the socalled flash-lag effect, also offers no good explanation. Nijhawan (1994) demonstrated a sizable flash-lag effect for frontoparallel motion. He presented a line in the frontoparallel plane, rotating around its center at 15 to $45 \mathrm{rpm}$. The center segment of the line was illuminated continuously, while the end segments were strobed. The ends appeared to lag behind the center part of the line. Nijhawan explained this effect in terms of how the visual system might compensate for the delay in the transmission of motion information from photoreceptors to higher visual areas of the cortex. He suggested that, in the case of moving objects, the visual system overcomes most of the transmission latency through extrapolation. Owing to the unpredictability of the stroboscopic event, the visual system cannot extrapolate the motion of the end segments; hence, they lag behind. Thus, the effect should disappear once the flash becomes predictable. However, we show that a predictable series of flashes continues to produce a sampling effect and can no longer be explained by the inability to anticipate the flash.

The situation is complicated by the somewhat different sampling effects found by Castet (1995), who examined linear frontoparallel motions. He created apparent motion by using an array of 32 LEDs, spaced $0.086^{\circ}$ from one another. Motion was produced by sequentially illuminating each LED in the series $\left(\Delta x=0.086^{\circ}\right)$ or by illuminating every second $\left(\Delta x=0.17^{\circ}\right)$ or every third $\left(\Delta x=0.26^{\circ}\right)$ LED. The more coarsely sampled motion appeared faster so long as the object motion was slow (2-4 deg/sec) and the stimulus onset time was short $(1-40 \mathrm{msec})$; the spatial interval of the LEDs had minimal effect at higher speeds $(8 \mathrm{deg} / \mathrm{sec})$ and longer onsets $(80 \mathrm{msec})$. Castet explained his findings in terms of a pooling of broadly tuned temporal filters.

The discrepancies between these findings and those of Nijhawan (1994) could result from several factors. First, the linear motions Castet (1995) employed may be easier to extrapolate than Nijhawan's rotating stimuli. Furthermore, Castet's paradigm required observers to compare the current stimulus with one previously seen; thus, memory encoding may have influenced the results.

To broaden the search for an explanation of the sampling effect, we investigated to what extent predictability is an issue, whether the sampling effect generalizes to motion in depth, and whether sampling might be an artifact of the computer-generated stimuli that are typically used in the relevant studies.

\section{A Time-to-Contact Paradigm to Study Motion-in-Depth Sampling}

There is reason to assume that the sampling effect generalizes to perceptual events outside the frontoparallel plane. Although the transience of objects does not seem to interfere with form perception (Dosher, Landy, \& Sperling, 1989; Todd, 1985), flickering targets appear to be farther away from the observer than nonflickering ones (Wong \& Weisstein, 1984). The flickering targets also appear smaller, which suggests that the visual pathways for both motion and form are affected by flicker (Miller \& Patterson, 1995). Transient stimuli also seem to disrupt size and space constancy (Macknik, Fisher, \& Bridgeman, 1991; Rogowitz, 1984). Rogowitz found that size constancy, which is usually highly robust during relative motion between target and observer, breaks down under stroboscopic illumination (ranging from 1 to $32 \mathrm{~Hz}$ ); the strobed object appeared to grow in size as it slowly approached the observer. This finding is consistent with results that targets flickering at rates of around $6 \mathrm{~Hz}$ are perceived as farther in depth than are continuously lit targets (Wong \& Weisstein, 1984). Miller and Patterson relate this effect to an underestimation of size owing to strobe lighting. However, the stationary size illusion caused by strobe light is quite small (about 1\% perceived shortening of a flashed line) and may, therefore, have little bearing on Rogowitz's findings.

Spatial relations are perceived rather differently in frontoparallel and sagittal perspectives. Typically, significant sagittal compression effects are found, resulting in large distances being grossly underestimated (Loomis, Da Silva, Philbeck, \& Fukusima, 1996). These effects persist in pic- 
tures as well as in real-world viewing (Hecht, van Doorn, $\&$ Koenderink, 1999). Such spatial compression suggests that arrival times may be underestimated for objects that move in depth away from or toward the observer. And, in fact, observers often underestimate the TTC of approaching objects, as compared with lateral motion (Schiff \& Oldak, 1990). Thus, to fairly compare motion sampling in the frontoparallel and the sagittal planes, a paradigm has to be found that allows observers to produce similarly accurate motion judgments for both cases. We chose TTC as an ideally suited methodology.

Under normal viewing conditions, observers successfully avoid obstacles, presumably because they pick up visual information about the TTC and time to passage (TTP) of oncoming targets. For instance, Lee and his colleagues examined intercept timing behavior in birds (Lee \& Reddish, 1981) and humans (Lee, 1976; Lee, Young, Reddish, Lough, \& Clayton, 1983) and concluded that observers exploit an optical variable that directly specifies arrival time (tau). Tau capitalizes on the relative rate of optical expansion undergone by the retinal image of the approaching object.

Very short exposure time to a moving object should, in principle, be sufficient to make a more or less accurate TTC estimate. That is, TTC estimates based on discontinuous viewing should not be inferior to those based on continuous viewing, provided the last sample presented is of sufficient duration to provide the basis for a TTC estimate. Observers are often able to make reliable and fairly accurate TTC/TTP judgments so long as approach velocity is constant and the object is continuously visible before the beginning of an extrapolation period (e.g., Kaiser \& Mowafy, 1993; McLeod \& Ross, 1983). However, it has been shown that observers' TTC estimates are sometimes compromised even under good viewing conditions and that observers might use simpler cue-based strategies, rather than deriving tau (Tresilian, 1993, 1994; Wann, 1996). For example, object size biases TTC judgments (DeLucia, 1999; DeLucia \& Warren, 1994; Michaels, 2000; van der Kamp, Savelsbergh, \& Smeets, 1997; but see Regan \& Vincent, 1995). Also, the direction of the approach trajectory has an effect on perceived TTC, which it should not (Schiff \& Oldak, 1990). Stewart, Cudworth, and Lishman (1993) further argued that at far distances, observers perceive size, distance, and velocity/acceleration separately and then integrate them, rather than perceiving TTC directly via tau, as is possible at closer distances (but see Stewart, Cudworth, \& Lishman, 1997; Tresilian, 1997).

In sum, as perceptually smooth as frontoparallel sampled motion may be, it is less informative than continuous motion. Since phenomenal reports of speed tend to be inaccurate, and since very different methodologies have been previously used in the context of sampled motion, we applied an arrival time paradigm to study the sampling effect. Using this TTC judgment paradigm, we explored whether temporal sampling has a negative impact on the judging of motion in depth. A variety of sampled motions were explored in computer-simulated scenarios (Experiment 1). Background effects were explored in Experiment 2. Finally, to assess whether judgment degradation resulted from artifacts associated with computer-generated displays, we replicated the sampling effect with natural objects (Experiment 3 ).

\section{EXPERIMENT 1 Simulation of Time-Sampled Approach}

This experiment was designed to assess whether time sampling has negative effects on arrival time judgments of motion in depth. To answer this question, we used a standard TTC paradigm. We simulated a target (against a background of fixed stars) moving toward the observers on a collision course (TTC) or a passage course (TTP). The stimulus was always visible for $5 \mathrm{sec}$; then, observers had to extrapolate the target's motion and indicate arrival time by pressing a button. When the motion was sampled, the last sample coincided with the last visible position of the continuous case. Thus, according to tau theory (Lee, 1976), observers should be equally accurate in both cases. However, if the frontoparallel results generalize to the three-dimensional (3-D) approach, we expect longer judged TTCs for sampled motion. Also, if our motion extrapolation mechanism can exploit the information provided by a regular rhythm (as is typical for hazard lights or aircraft position lights), TTC estimates should be affected only by irregular time sampling. Experiment 1 therefore tested continuous illumination, regularly sampled illumination, and randomly sampled illumination.

\section{Method}

Observers. Eight male observers were recruited by an on-site organization at NASA Ames Research Center. They ranged in age from 29 to 38 years and had normal or corrected-to-normal vision. Most attended local colleges and universities. They were paid for their participation.

Apparatus and Stimuli. The stimuli were generated on a Silicon Graphics Onyx RE2 workstation with a resolution of 1,280 $\times 1,024$ pixels and a refresh rate of $72 \mathrm{~Hz}$ (noninterlaced). The animation update rate was 72 frames/sec. An Electrohome RGB rear projection system was used to display the animation on a large screen $(244 \times$ $183 \mathrm{~cm}$ ). The observer was seated $300 \mathrm{~cm}$ away from the screen in a height-adjustable chair to align his line of sight at the center of the display screen; the display subtended $44.2^{\circ}$ of visual angle horizontally by $33.9^{\circ}$ vertically.

The stimulus consisted either of a multicolored cube that maintained a stable orientation as it approached the observer or of a constantsize red dot. The cube had a luminance of approximately $5 \mathrm{~cd} / \mathrm{m}^{2}$. The otherwise black background contained approximately 1,000 single-pixel white dots (i.e., fixed stars). As is depicted in Figure 1, a viewing volume was defined to be 7,500 graphical units (gu) deep; given the approach speeds of the target, arrival times ranged from 6 to $10 \mathrm{sec}$ after onset of motion. Since familiar objects were not depicted in the scene, absolute distances were not defined (i.e., graphical units were computed on an arbitrary scale). The projected size of the target cube grew according to the geometry of a polar projection as it approached. The single-dot target maintained the same projected size ( 2 pixels) throughout the animation sequence. Initial target depth varied between 3,000 and 4,000 gu. Target approach velocities were 
chosen randomly on each trial, ranging from 400 to $500 \mathrm{gu} / \mathrm{sec}$. Thus, on an average trial, the projected size of the cube increased from approximately $0.5^{\circ}$ to about $5^{\circ}$ of visual angle. This was important, since target velocity has effects on TTC judgments (Sidaway, Fairweather, Sekiya, \& McNitt-Gray, 1996). The observer and the background starfield remained stationary, whereas the target was simulated to approach the observer. Each trial consisted of an animated sequence that terminated after $5 \mathrm{sec}$. Observers then had to extrapolate the target motion and judge when the object would transition the observer's eye plane. Extrapolation times until contact/passage averaged $2.7 \mathrm{sec}$, ranging from 1.2 to $4.5 \mathrm{sec}$.

Design. A three-factor, within-subjects design was used. The first factor, type of transience, had nine levels. Targets were continuously visible, flashing regularly, or flashing randomly. In the regular conditions, targets flashed at $4 \mathrm{~Hz}$ or at $1 \mathrm{~Hz}$, crossed with ontimes (i.e., duty cycles) of one and four frames (14 and $56 \mathrm{msec}$, respectively). In the random sampling condition, average frequencies and ontimes were equated with the regular conditions, but the flashes were randomly distributed over the visible 5-sec interval. A minimum stimulus onset asynchrony of $100 \mathrm{msec}$ was always maintained. The nine levels of transience are summarized in Table 1. The update rate of the display and its refresh rate were both $72 \mathrm{~Hz}$. (Thus, strictly speaking, motions were always time sampled. However, this update rate is sufficiently high that we operationalize it to be continuous.) The position of the targets was updated during the duty cycle, except in those cases in which it did not exceed one refresh cycle $(14 \mathrm{msec})$. In that case, time sampling was effectively discrete.

The second factor, stimulus shape and offset, had five levels: a constant-size dot with 100-gu lateral offset, a constant-size dot with 200-gu lateral offset, an expanding cube centered on the line of sight, a cube with 100-gu lateral offset, and a cube with 200-gu lateral offset. Varying lateral offset partially decorrelates target distance and initial angular offset (Kaiser \& Mowafy, 1993). Nested within this factor and irrelevant to the analysis, the dot and the cube were presented randomly to the left or to the right of the line of approach (TTP), except for those cases in which the cube approached along the line of sight (TTC). No TTC condition was presented for the dot, since the lack of local expansion negates TTC information and would render the task meaningless.

The third factor, initial target depth, had six levels. Initial target depth ranged from 3,000 to 4,000 gu in increments of $200 \mathrm{gu}$. In addition, the constant target velocity varied randomly from trial to trial between 400 and $500 \mathrm{gu} / \mathrm{sec}$. All three factors were fully crossed, resulting in a total of 270 trials, which were presented to each observer in one single block with different random orders.

Procedure. The observers were seated in a dark room, $3 \mathrm{~m}$ from the large screen display, so that their line of sight was centered on the screen. It was explained that only the target object (a red dot or a cube) would appear to move toward the observer and that the display would terminate after $5 \mathrm{sec}$. An eyepatch was worn over the observer's nondominant eye to minimize interference from inconsistent binocular depth cues. To emphasize the visual nature of the task, the observers were instructed to imagine the continuation of the motion and to click the left button of a three-button mouse at the point in time at which the target would hit or pass their eye plane. The next trial started automatically after a 4 -sec pause. The observers received approximately 15 practice trials, using somewhat different target motion parameters. To help the observers calibrate their judgments, feedback (signed TTP/TTC error in milliseconds) was given during the practice session, but not during the experimental trials.

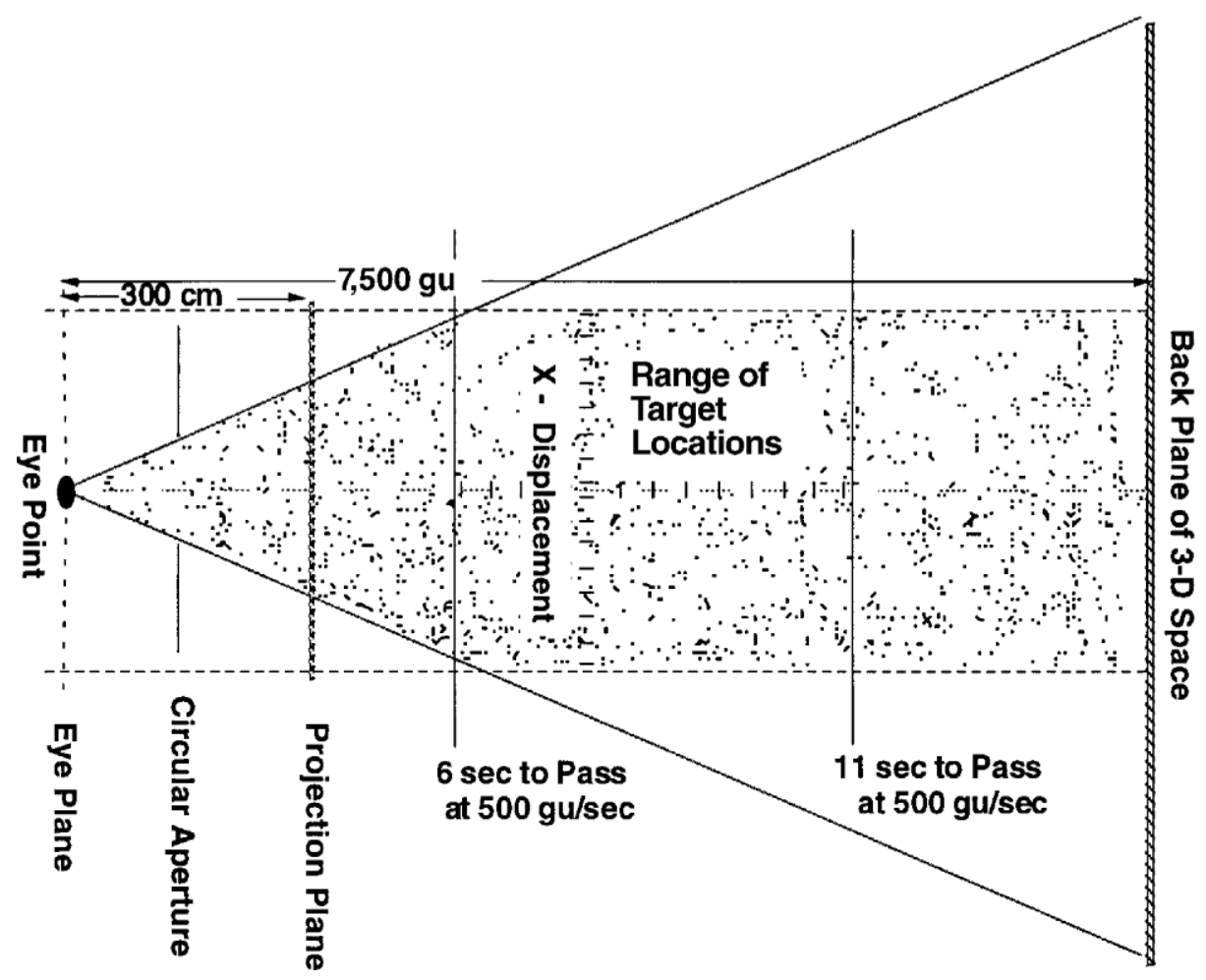

Figure 1. Schematic drawing of the simulated viewing volume used in Experiments 1 and 2. Targets were positioned in the rear and center portion of the volume. Their positions differed in depth as well as in $x$-displacement. The dotted line originating at the eye point represents the track vector. The field of view in Experiment 1 subtended $44.2^{\circ}$ horizontally by $33.9^{\circ}$ vertically. 
Table 1

The Factor of Transience as Varied in Experiment 1 (Computer Simulation)

\begin{tabular}{clcc}
\hline Level & Sampling & Frequency $(\mathrm{Hz})$ & Duty Cycle $(\mathrm{msec})$ \\
\hline 1 & continuous & 72 & - \\
2 & regular & 1 & 14 \\
3 & regular & 1 & 56 \\
4 & regular & 4 & 14 \\
5 & regular & 4 & 56 \\
6 & random & 1 & 14 \\
7 & random & 1 & 56 \\
8 & random & 4 & 14 \\
9 & random & 4 & 56 \\
\hline
\end{tabular}

Note-During the duty cycle, the stimulus was updated every refresh cycle (14 msec).

\section{Results}

Observer responses more than $5 \mathrm{sec}$ before or after the actual TTC/TTP were considered accidental and were excluded from further analysis. (This value corresponded to more than three standard deviations; excluded trials accounted for only $1.2 \%$ of the data.) Repeated measures analyses of variance (ANOVAs) were performed on TTC and TTP errors. Error was defined as the difference between optically specified TTC and judged TTC. Constant error (i.e., positive error values for early responses and negative values for late responses) was the dependent measure. For a first analysis, only TTP trials were analyzed. As is depicted in Figure 2, a large effect $[F(1,7)=$ $12.57, p=.0094$ ] was found for stimulus type; dot targets led to a much greater overestimation of arrival time than did cube targets. Motion sampling also produced a large main effect $[F(2,14)=27.97, p=.0001]$. Regular and ran- dom time sampling did not differ significantly in TTP judgments, but continuous motion led to significantly shorter TTP estimates than did randomly $[F(1,7)=33.64$, $p=.0007]$ and regularly $[F(1,7)=29.70, p=.001]$ sampled motion. Target type did not interact with motion sampling.

To understand how well the observers judged TTCs with respect to the true value that was optically specified, we tested whether the raw errors differed from 0 . The observers were accurate when presented with continuous dots or with sampled cubes, even by this least conservative measure. That is, according to a plain $t$ test, their TTC/ TTP estimates did not significantly differ from the correct value. TTCs for continuous cubes were systematically underestimated [smaller than specified value; $t(141)=3.15$, $p=.002]$, whereas TTPs for sampled dots were consistently overestimated $[t(374)=-9.69, p<.001]$. The most conservative test, based on averaged results, yielded significant timing errors only for dot targets $[t(7)=-2.55$, $p=.038$, for random sampling, and $t(7)=2.21, p=.063$, for regular sampling]. However, absolute errors should not be overinterpreted, since the baseline of TTC judgments can easily be shifted and, in the present experiment, the observers seem to have chosen sampled cubes as their baseline. We defer this issue to the General Discussion section and focus on the relative differences here.

Duty cycle made a difference only for $4-\mathrm{Hz}$ stimuli. Lengthening ontime from one frame $(14 \mathrm{msec})$ to four frames $(56 \mathrm{msec})$ did not significantly impact TTP judgments for 1-Hz stimuli but did lead to smaller errors for 4$\mathrm{Hz}$ stimuli $[F(1,7)=8.75, p=.0211]$; the judgments for the one-frame trials were, on average, late by $194 \mathrm{msec}$ versus $32 \mathrm{msec}$ for the four-frame trials. Duty cycle did not interact with sampling (random vs. regular). An analy-

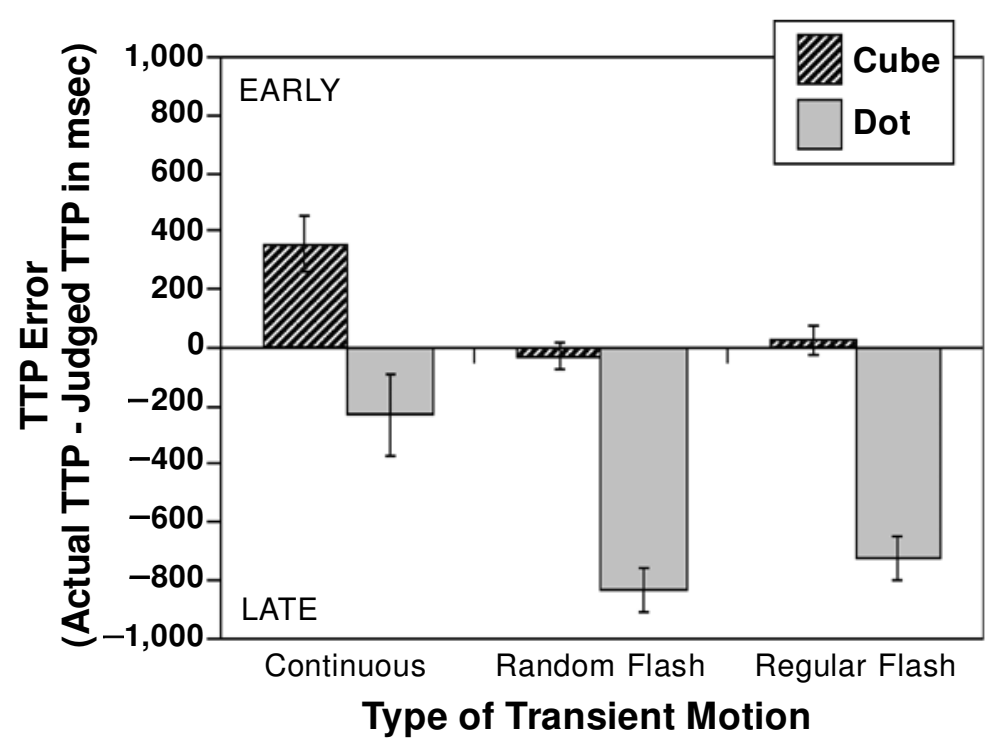

Figure 2. Mean response times collected in Experiment 1. Random and regular time sampling produced equal overestimation. Large objects were judged to arrive earlier than small objects. Error bars represent standard errors of the mean. TTP, time to passage. 
sis within cube stimuli did not yield any significant difference in accuracy between TTC and TTP trials.

\section{Discussion}

The sampling effect for motion in the frontoparallel plane was replicated for motion in the sagittal plane. Across all stimuli, sampling motion led to arrival time judgments consistent with slower estimations of target velocity. In addition to this main effect, the cube targets produced shorter (and generally more accurate) TTP judgments than did the constant-size target stars. Two interpretations for this result are possible. First, the cube stimulus provided richer temporal information because TTP was specified by local object expansion as well as by global object displacement rates from the line of sight. This redundancy of information could have improved accuracy. Second, luminance and size was much smaller for dot targets than for the cubes. This could have led to a baseline shift for TTP estimates in the observed direction (cf. DeLucia \& Warren, 1994) and, in Helson's terms, to a change in adaptation level (Helson \& Bevan, 1967). Moreover, the dot target can be considered to be providing conflicting cues about its distance, since it fails to expand (see, e.g., Gray \& Regan, 1999). Thus, results should most properly be evaluated in relative terms. Temporally sampled dot stimuli, as well as sampled cube stimuli, lead to longer TTP (and, for cube stimuli, TTC) judgments than do their continuous counterparts.

Errors that have been noted in ball catching tasks with intermittent occlusion (Elliot, Zuberec, \& Milgram, 1994) and kinematic changes in the timing of grasping action (Savelsbergh, Whiting, Pijpers, \& van Santvoord, 1993) are consistent with our findings. Velocity underestimation owing to time sampling could have led to delayed motor responses, which, in turn, resulted in errors and strategy changes in timing of the catching.

Surprisingly, the nature of the time sampling (regular vs. random intervals) had no appreciable effect on arrival time judgments. Lengthening the duty cycle, on the other hand, did reduce the error that was due to time sampling for the $4-\mathrm{Hz}$ stimuli. This may be suggestive of a pooling mechanism that is imperfect at integrating motion information across larger temporal gaps. This possibility will be examined more fully in Experiment 2.

\section{EXPERIMENT 2 Observer Motion Versus Object Motion}

This experiment was designed to serve two functions. First, total viewing time (aggregated ontime) was perfectly confounded with sampling in Experiment 1. To decouple the two, we varied the number of samples and their length independently in Experiment 2. Also, a more thorough analysis of sampling rates can test for a correlation of TTC overestimation and decreases in the sampling rate. Second, in Experiment 1, the target moved in front of a stationary background (simulating a stationary observer).
The computationally equivalent case of a moving observer (and a stationary target) might enhance observers' ability to interpolate between flashes. That is, a moving background of stars might give additional perceptual support of the optical flow velocity and, thus, help specify TTC. A moving background is also suggestive of a cause for the sampling outside of the stimulus, whereas sampling of the target alone suggests a cause in the scene or even in the target. To determine whether ongoing perceptual support facilitates TTC judgments, we examined the effect of sampling the entire scene versus only the target.

As in Experiment 1, the visual stimuli were computer generated. The same viewing volume containing 1,000 white stars was employed (see Figure 1). In this experiment, the stars could move in synchrony with the target cube (suggesting sagittal observer motion toward the target), or they could remain stationary (suggesting target motion toward the stationary observer). In all cases, the entire display was turned off for the extrapolation period. In all trials, TTP judgments of a cube stimulus on passage course was made.

\section{Method}

Observers. Nine NASA Ames employees (4 male, 5 female) volunteered to participate as observers. They ranged in age from 26 to 42 years and had normal or corrected-to-normal vision. None had prior knowledge of the purpose of the study or had participated in the previous experiment.

Apparatus and Stimuli. As in Experiment 1, a Silicon Graphics Onyx RE2 workstation with a display resolution of $1,280 \times 1,024$ pixels and a graphics animation update rate of $72 \mathrm{~Hz}$ was used. The observers sat in a darkened room, with their line of sight centered on a 20 -in. diagonal $(38 \mathrm{~cm}$ horizontal $\times 29 \mathrm{~cm}$ vertical) display screen. To achieve a visual angle equivalent to that in Experiment $1\left(44.2^{\circ} \times\right.$ $33.9^{\circ}$ ), the observer's eye point was set $50 \mathrm{~cm}$ from the monitor. The target for the TTP judgments was a 3-D multicolored cube. As before, on an average trial, the projected size of the cube increased from approximately $0.5^{\circ}$ to $5^{\circ}$ of visual angle during its visible trajectory, varying with initial target depth and velocity.

Design. Eight stimuli differing in sampling rate and ontimes were created. At 14-msec ontime (corresponding to one display frame), sampling rates were $1,2,4,8,18$, and $72 \mathrm{~Hz}$. For control purposes, a $1-\mathrm{Hz}$ stimulus with a $225-\mathrm{msec}$ ontime and a $4-\mathrm{Hz}$ stimulus with a 56-msec ontime were added. In terms of the total time the stimulus was visible per trial, these cases matched the $18-\mathrm{Hz}$ stimulus. The transience conditions are summarized in Table 2 .

These stimuli were fully crossed with the following factors. The first was the extent of the visual scene that was time sampled (two

Table 2

The Factor of Transience as Varied in Experiment 2 (Computer Simulation)

\begin{tabular}{clcc}
\hline Level & Sampling & Frequency $(\mathrm{Hz})$ & Duty Cycle $(\mathrm{msec})$ \\
\hline 1 & continuous & 72 & - \\
2 & regular & 18 & 14 \\
3 & regular & 8 & 14 \\
4 & regular & 4 & 14 \\
5 & regular & 2 & 14 \\
6 & regular & 1 & 14 \\
Control & regular & 1 & 225 \\
Control & regular & 4 & 56 \\
\hline
\end{tabular}


levels). Either the entire scene flickered or only the target flickered. Second, the starfield could move in synchrony with the target (suggestive of observer motion), or it could be stationary (suggestive of target motion).

Third, to ensure sufficient variability in TTP, the targets were presented at starting locations corresponding in depth to $3,000,3,500$, and 4,000 gu, crossed with displacement to the left or the right of the line of sight by 100 and by 200 gu (six levels). Nested within this factor, target (or target and starfield) velocities varied randomly between 400 and $500 \mathrm{gu} / \mathrm{sec}$. Thus, TTPs ranged from 1 to $5 \mathrm{sec}$ after display termination. This created a total of $192(8 \times 2 \times 2 \times 6)$ trials, which were presented in different random orders to each observer.

Procedure. The procedure was the same as that used in Experiment 1 . All the stimuli were viewed monocularly with the observer's preferred eye. As before, the stimulus display was visible for $5 \mathrm{sec}$, then disappeared entirely, as did the background. As in Experiment 1, times until hypothetical passage averaged $2.7 \mathrm{sec}$ (ranging from 1.2 to $4.5 \mathrm{sec}$ ). The observers pressed a mouse button to indicate the instant of passage. To familiarize the observer with the task, 15 practice trials were given. Feedback in signed TTP error in milliseconds was given only during these practice trials. Short breaks were taken after every 50 trials (approximately every $10 \mathrm{~min}$ ).

\section{Results}

As before, repeated measures ANOVAs were performed. As is shown in Figure 3, a main effect for sampling rate was found $[F(7,56)=7.08, p<.0001]$. Generally, the lower the sampling rate, the later the judged TTP. For the single-frame ontime stimuli, a high regression coefficient between $\log ($ hertz) and TTP error (performed on cell means) was found $\left(r^{2}=.84, p=.016\right)$. Thus, the relation between TTP overestimation and the log sampling rate approached linearity. All sampling conditions produced TTP

\section{Type of transient motion ( $\mathrm{Hz}$ \#on-frames)}

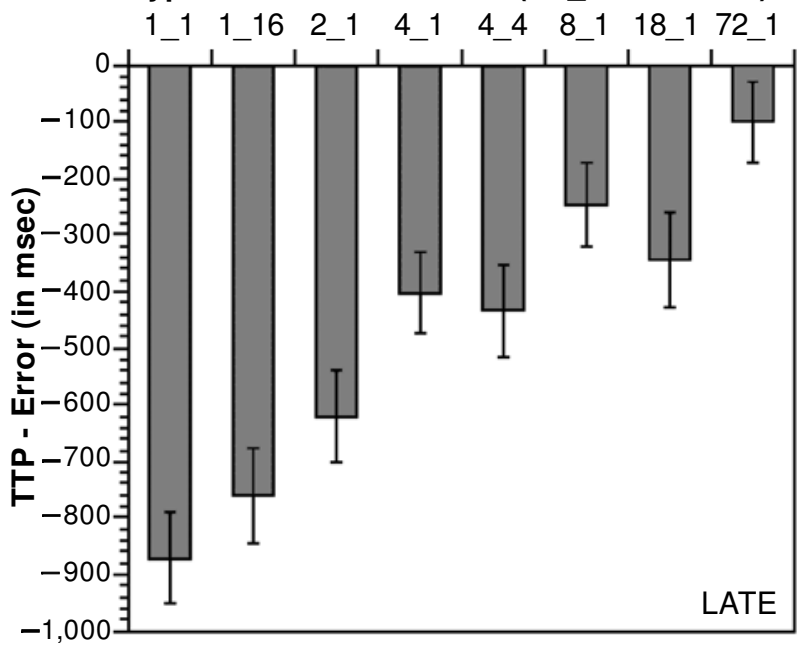

Figure 3. Average time-to-passage (TTP) errors as a function of sampling rates and ontimes in Experiment 2. A negative linear relationship between the log sampling rate and TTP overestimation was found. Surprisingly, the ontime variations produced no significant effects. Error bars represent standard errors of the mean. judgments that overestimated the specified value ( $t$ values ranging from 3.1 to 8.1 ), whereas continuous trials were judged accurately (i.e., no significant differences from true value).

A main effect for starfield motion was found; its presence led to overall smaller TTP errors $[F(1,8)=9.98, p=$ .013 ]. However, as can be seen in Figure 4, this reduction in error for the moving starfield occurred only for sampling rates of $8 \mathrm{~Hz}$ and below. This interaction between sampling rate and starfield motion was statistically significant $[F(7,56)=3.94, p=.0033]$. That is, continuous $(72-\mathrm{Hz})$ and $18-\mathrm{Hz}$ motion did not demonstrate the moving starfield effect. Also, a trend was found indicating that the facilitating effect of starfield motion at low sampling rates $(1 \mathrm{vs} .72 \mathrm{~Hz})$ was strongest for the long duty cycle of 16 frames [222 msec; $F(1,8)=3.53, p=.082]$. In other words, the observers benefited less from the moving starfield in cases in which low sampling rates were combined with short ontimes. The sampling mode (i.e., whether the entire visual field or just the target flashed on and off) produced no significant main effect or interactions.

\section{Discussion}

The effect of time sampling on TTP overestimation was replicated. For the tested range of sampling rates of between 18 and $1 \mathrm{~Hz}$, the observers overestimated TTP (corresponding to an underestimation of target velocity). Overestimation was larger with lower sampling rates. Moreover, the suggestion of observer motion (accomplished by synchronized target and background movement) ameliorated the sampling effect. That is, the moving starfield had a facilitating effect and seemed to support the observers' ability to interpolate and/or extrapolate the target's motion. This facilitating effect of background motion was not evident when the display was sampled at rates of $18 \mathrm{~Hz}$ and above. In these cases, perceptually smooth and continuous motion was experienced.

One possible explanation for the time-sampling effect rests on the assumption of a spatial pooling mechanism, which would cause a misjudgment of target position (Helson \& Bevan, 1967). That is, the position assigned to the moving target when last visible could correspond, not to the position of the final flash, but rather to the location between the final two flashes. Such an extreme position would no longer be compatible with a tau explanation. Nonetheless, we tested the assumption of such mislocalization with the present data. Modified TTP values were calculated by subtracting the time interval corresponding to half the distance between the last two flashes.

The average TTP errors based on these modified TTP values are shown in Figure 5. (Note that modified TTP values remained unchanged for continuous stimuli, whereas they were largest for stimuli sampled at $1 \mathrm{~Hz}$.) The regression between $\log ($ hertz) and TTP error performed on cell means for short ontimes revealed a less significant relationship, as compared with the unmodified analysis $\left(r^{2}=.635, p=.0576\right)$. However, an ANOVA on the mod- 


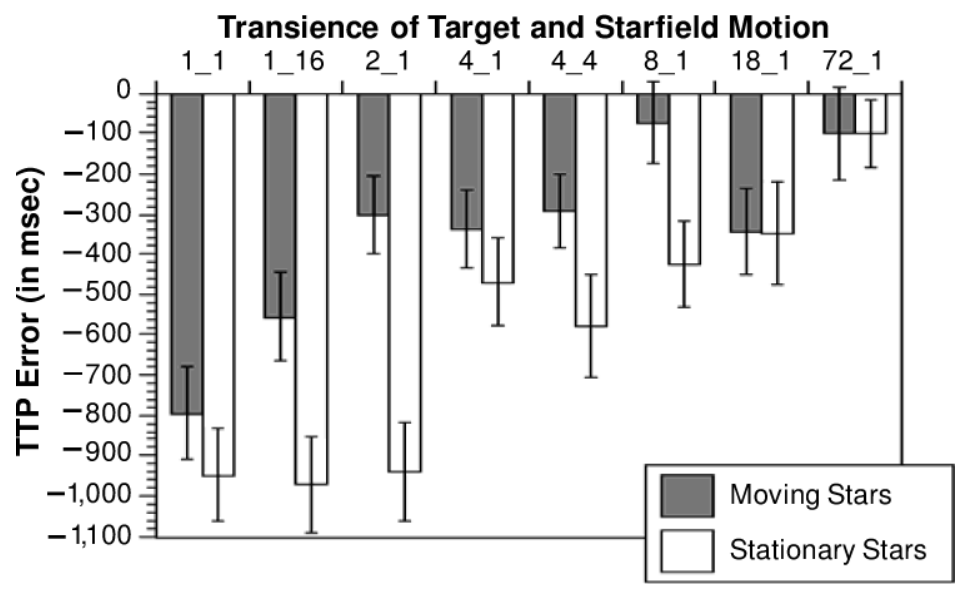

Figure 4. Average time-to-passage (TTP) errors by starfield motion in Experiment 2. The starfield could move synchronized with the target, suggesting sagittal observer motion, or it could be stationary, suggesting target motion toward the observer. Error bars represent standard errors of the mean.

ified data still produced a main effect for time sampling to increase TTP judgments $[F(7,56)=3.18, p=.0067]$. The much attenuated effect suggests that even an extreme mislocalization of the stimulus cannot explain the timesampling effect.

\section{EXPERIMENT 3 \\ Replication With Analogue Stimuli}

It is conceivable that the computer simulations produced the observed sampling effects only because of artifacts of the display (e.g., limited frame rate). Even during the visible periods, the stimulus was always sampled; it was refreshed at $72 \mathrm{~Hz}$. This flicker might interfere with long-range motion integration and proper updating of arrival time estimates. Motion blur algorithms attempt to avoid visible aliasing effects that arise when fast motion is simulated, but they cannot replace viewing of real objects. Other problematic factors were cues to planar motion produced by the two-dimensional screen, its limited resolution, and the knowledge of being in a very small room. In Experiment 3, we introduced real physical objects and a realistic (albeit mimed) catching task. Unfortunately, when moving to more ecological stimuli, new constraintsemerged that prevented the creation of stimuli with the ecological setup that were retinally identical to the computer-based studies. Most important, extrapolation times had to be much shorter because of the spatial limitations of the motion track. Viewing times were adjusted accordingly to leave a roughly similar ratio of viewing to extrapolation time. Fortunately, if shortened viewing times introduce a bias, it will be on the conservative side, making it harder to find the sampling effect: If the sampling effect is caused by a cognitive mechanism that distorts TTC information during the extrapolation period, short extrapolation times are less likely to suffer from such corrections. If this is the case, or if the ecological viewing situation provides superior information to that from the computer simulations, the sampling effect should disappear. If, on the other hand, the sampling effect reflects a basic mechanism, it should persist.

In sum, in this experiment, we examined whether the sampling effect is sufficiently robust to occur with a real object, a natural motor response, and short extrapolation times. Using a subset of the temporal sampling set from Experiment 1, we measured the hand movements of participants asked to execute a grasping movement to catch a passing ball.

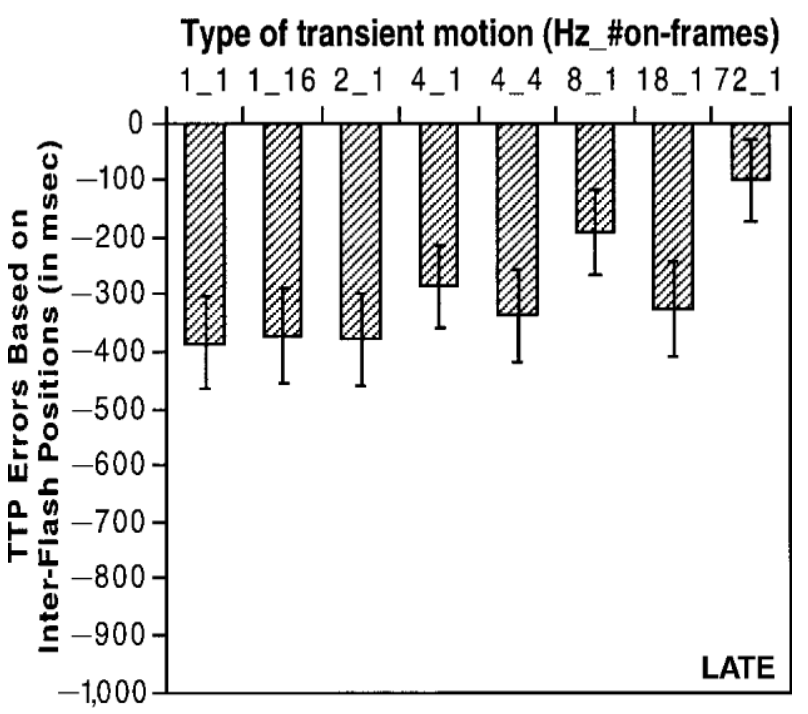

Figure 5. Average time-to-passage (TTP) errors from Experiment 2 modified to reflect a pooling mechanism that mislocates the target to be between the last two visible flashes before it disappears. Error bars represent standard errors of the mean. 


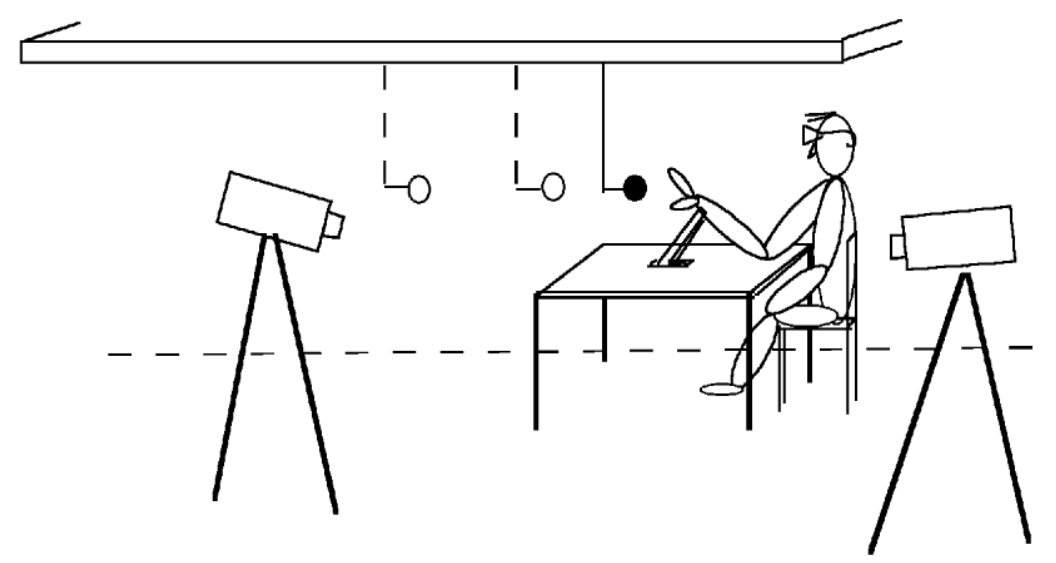

Figure 6. Schematic drawing of the ball transport apparatus used in Experiment 3. The movement of an approaching ball was computer controlled and synchronized to shutter glasses worn by the observer. Thus, the ball was visible only during its straight approach path, sometimes continuously, sometimes time sampled.

\section{Method}

Observers. Eight graduate students and 2 employees of the Faculty of Human Movement Sciences (5 male, 5 female) at the Vrije Universiteit of Amsterdam served as observers. They ranged in age from 19 to 49 years, had normal or corrected-to-normal vision, and were paid for their participation.

Apparatus and Stimuli. A ball, which was painted with luminous paint and had a diameter of $6 \mathrm{~cm}$, was used as the approaching object. The ball's paint was charged for $10 \mathrm{sec}$ before each trial with a 600-W light bulb. During the trials, only the luminous ball was visible in an otherwise darkened room.

The ball was presented using the ball transport apparatus (BallTrAp), which is depicted in Figure 6. The BallTrAp (see van der Kamp et al., 1997, for a detailed description of this apparatus) consisted of a wooden box $(305 \times 110 \times 15 \mathrm{~cm})$ mounted along the ceiling. It contained two aluminum wheels $(80 \mathrm{~cm}$ in diameter), with their centers mounted $210 \mathrm{~cm}$ apart. A rubber belt $(651 \mathrm{~cm})$ connected the two wheels. Fixed to the belt was a little trolley with an aluminum rod $(58.5 \mathrm{~cm}$ in length), at the end of which the ball was attached with Velcro. One wheel was driven by a Micron MT30r4-58 ServoMotor (maximal torque, 3.5 NM; maximal speed, 2,500 rpm). The Servo-Motor was controlled by a Galil DMC-700 Motion Controller, which received commands for the distance, velocity, and acceleration of the target ball from a PC. The BallTrAp had a support structure, so that the wooden box was positioned $155 \mathrm{~cm}$ above the floor.

The participant sat in a chair with his or her right arm and wrist resting on an adjustable armrest, which held the lower arm fairly rigid while giving freedom to movements of the hand. The participant's arm was positioned $50 \mathrm{~cm}$ left of the track of the ball at the end of its $210-\mathrm{cm}$ straight path, so that the ball passed to the right side of the participant's hand. The distance between eyes and hand was approximately $40 \mathrm{~cm}$.

The observers wore liquid crystal spectacles (Plato System P-1), which were also controlled by the $\mathrm{PC}$, thereby synchronizing vision with the ball's position. Vision was monocular (right eye). To mask sound cues from the BallTrAp, music was played through a set of headphones; instructions were likewise delivered over the headphones.

Grasping movements served as the dependent measures and were encoded using a 3-D SELSPOT monitoring system, which consisted of two SELCOM 413-3 cameras. By means of this data registration system, the position of four infrared light sources (LEDs) was monitored. The light sources consisted of LEDs fixed to the end of the target's mounting rod, to the first thumb and the first index finger phalanx, and to the medial side of the hand (the musculus adductor pollicis, caput transversum). The position signal was sampled at a frequency of $313.2 \mathrm{~Hz}$. The SELSPOT system was precalibrated at the start of the experiment to an accuracy of less than $3 \mathrm{~mm}$.

Design. All factors were varied within observers. Four constantapproach velocities were used $(0.25,0.5,1.0$, and $2.0 \mathrm{~m} / \mathrm{sec})$. These velocities were fully crossed with four sampling rates (continuous motion, $4 \mathrm{~Hz}$ with 28 -msec ontimes, $8 \mathrm{~Hz}$ with 28 -msec ontimes, and $4 \mathrm{~Hz}$ with 56-msec ontimes). For continuous motion trials, the ball was always visible for exactly $1 \mathrm{sec}$ during its straight approach trajectory toward the observer. The shutter glasses closed at the moment of passage (MoP) and remained shut thereafter. For time-sampled trials, the shutter glasses closed intermittently during the 1-sec period. The total ontime during which the stimulus was visible $(224 \mathrm{msec})$ was equated for the long $(56-\mathrm{msec}) 4-\mathrm{Hz}$ condition and the $8-\mathrm{Hz}$ condition. Ontime was $112 \mathrm{msec}$ for the short (28-msec) $4-\mathrm{Hz}$ condition. Extrapolation times, measured from the moment the shutter closed for the last time to MoP, were $124 \mathrm{msec}$ for the 4-Hz, 28-msec condition, $96 \mathrm{msec}$ for the $4-\mathrm{Hz}, 56-\mathrm{msec}$ condition, $74 \mathrm{msec}$ for the $8-\mathrm{Hz}, 28-\mathrm{msec}$ condition, and $0 \mathrm{msec}$ for the continuous cases. Each stimulus was repeated six times during the randomly ordered set, for a total of 96 trials. The levels of transience are summarized in Table 3. Sampling was continuous (i.e., the object continued to move during the duty cycle).

Procedure. The participants were instructed to hold the thumb in contact with the index finger at the start of every trial. The reconstructed 3-D positions of the thumb and index finger LEDs were fil-

Table 3

The factor of Transience as Varied in Experiment 3 (Real World)

\begin{tabular}{clcc}
\hline Level & Sampling & Frequency $(\mathrm{Hz})$ & Duty Cycle $(\mathrm{msec})$ \\
\hline 1 & continuous & - & - \\
2 & regular & 4 & 28 \\
3 & regular & 4 & 56 \\
4 & regular & 8 & 28 \\
\hline
\end{tabular}

Note-All flashing targets were sampled continuously-that is, they were in motion during their duty cycle. 


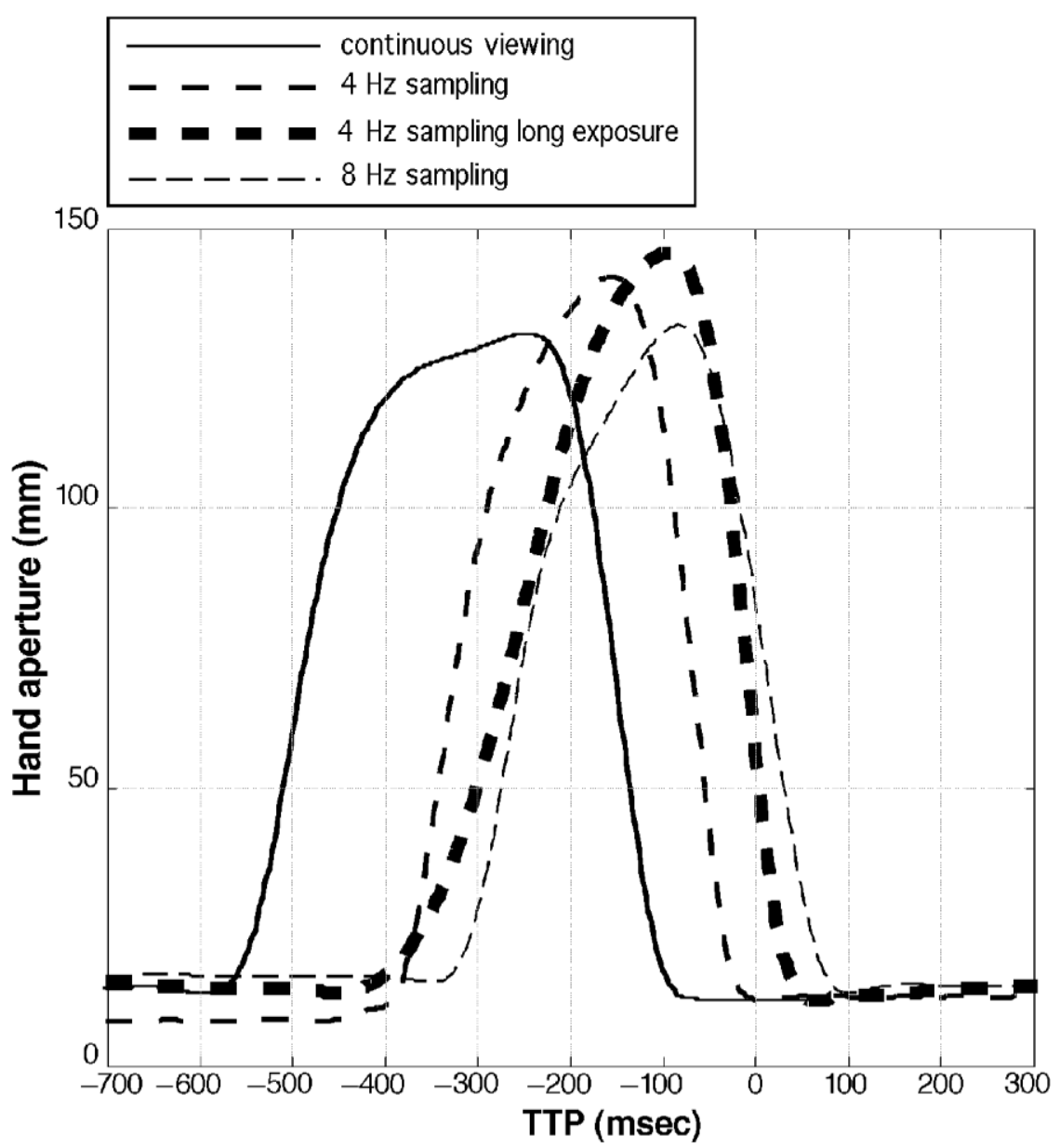

Figure 7. Recorded hand aperture data for sample trials of Experiment 3, which reflect the early responses typical for slow moving $(0.5 \mathrm{~m} / \mathrm{sec})$ targets. Time-position curves are plotted for all four time-sampling conditions. TTP, time to passage.

tered with a second-order Butterworth filter, with a cutoff frequency of $10 \mathrm{~Hz}$ (applied twice in order to negate phase shift). The experimenter explained that the approaching ball would always move toward the participant but would miss his or her right hand by the 50$\mathrm{cm}$ offset. Consequently, they would not actually catch the ball, but should execute a virtual grasp. (Actual grasping would have risked injury in trials with fast approach velocities.) The grasp was to be executed quickly; that is, the participant was encouraged to open his or her hand as late as possible and to make closing contact between the thumb and the forefinger when the ball was parallel to the palm.

\section{Results}

The kinematic characteristics of the grasping phase of the catch were determined as follows. First, the MoP $(\mathrm{TTP}=0)$ was defined as the moment at which the distance in the approach direction between the ball LED and the hand $L E D$ was zero. Adaptation of hand aperture was determined by calculating the distance between the thumb and the index finger. All timing measures were defined with respect to the MoP. Following earlier work of Savelsbergh, Whiting, and Bootsma (1991; Savelsbergh et al.,
1993), several dependent variables were used: moment of grasp onset, the time at which the hand began to be opened (i.e., when the participant started increasing thumb-index-finger distance); moment of maximal aperture, the time at which the distance between the thumb and the index finger was maximal and the hand started to close again; and moment of catch completion, the time of complete hand closure (i.e., when the thumb-index-finger distance reached its minimum).

Figure 7 shows sample timelines for hand aperture (thumb-index-finger distance), produced by one participant for four different trials (one from each sampling condition, all with approach velocities of $0.5 \mathrm{~m} / \mathrm{sec}$ ). In these particular examples, one can observe that completed hand closure occurred about $100 \mathrm{msec}$ before true TTP for continuous viewing and about $100 \mathrm{msec}$ after TTP for $8-\mathrm{Hz}$ sampled viewing.

Examining the three measures (grasp onset, maximal aperture, and complete closure of thumb and index finger), it is apparent that only closure is influenced systematically 


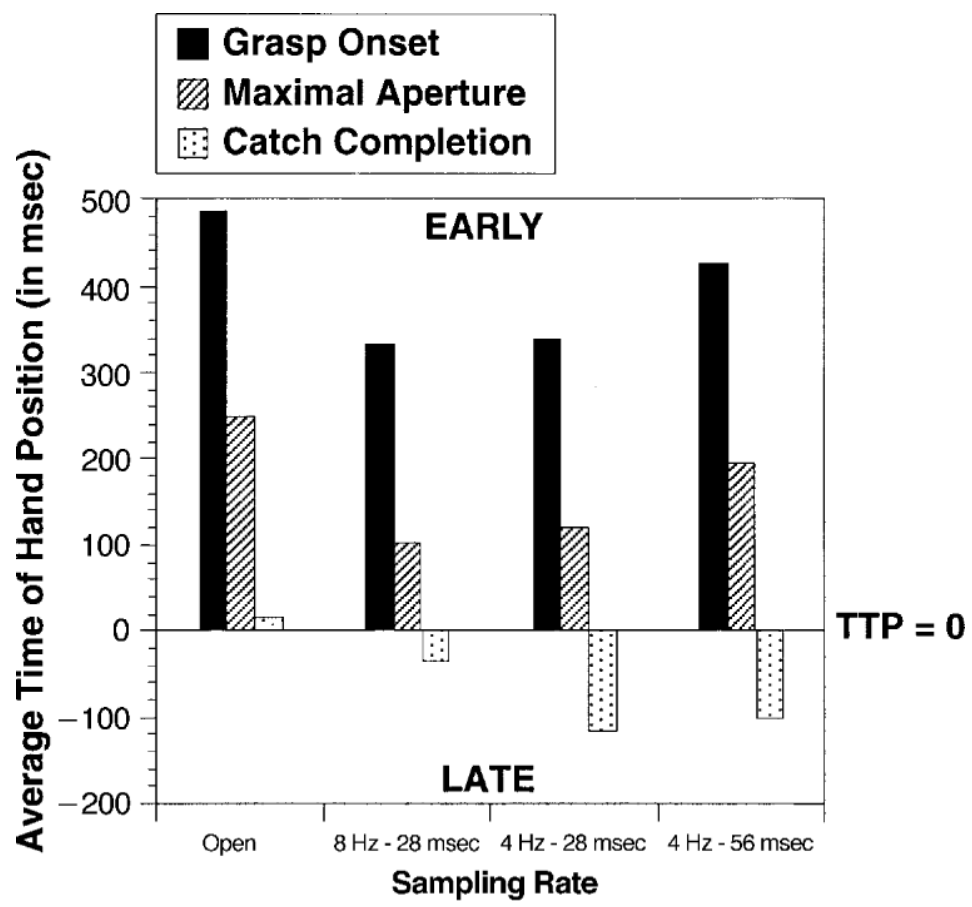

Figure 8. Average times for start of hand opening, maximal aperture, and hand closing in Experiment 3. Observers always tended to get ready $\sim 400 \mathrm{msec}$ before time to passage (TTP) - that is, not the whole action was delayed with time sampling, but only the closure of the fingers.

by the nature of the time sampling (see Figure 8 ). This is consistent with the instructions given; the participants always tended to prepare about $400 \mathrm{msec}$ before MoP. Thus, the entire action was not delayed because of time sampling: only the closure of the fingers was.

For each observer, the mean and standard deviation in each condition were calculated for the three dependent variables. A 4 (velocity) $\times 4$ (sampling rate) ANOVA, with repeated measures on both factors, was carried out on the average time-points of the end of the virtual grasp (catch completion). The $p$ values of all significance scores were Greenhouse-Geisser adjusted because of the relatively small number of trials.

Time sampling significantly delayed catch completion $[F(3,27)=50.14, p<.0001]$. Continuous motion was judged more accurately than $4-\mathrm{Hz}$ and $8-\mathrm{Hz}$ motion $[F(1,9)=21.82, p=.0012]$. No significant difference between the two types of $4-\mathrm{Hz}$ sampling was found. Sampling at $8 \mathrm{~Hz}$ led to smaller timing errors than did $4-\mathrm{Hz}$ sampling $[F(1,9)=89.15, p<.0001]$. A significantinteraction between velocity and sampling rate was also found $[F(9,81)=2.99, p=.0316]$. Separate $t$ tests for deviation of judged from actual TTP were significant only for the two $4-\mathrm{Hz}$ sampling rates $[t(9)=2.75$ and $2.51, p<.03]$. That is, for continuous viewing and $8-\mathrm{Hz}$ sampling, the observers made accurate judgments (as measured by catch completion), whereas they completed their catches too late with $4-\mathrm{Hz}$ sampling.
As is shown in Figure 9, average differences between complete hand closure and true TTP varied significantly as a function of approach velocity $[F(3,27)=9.39, p=.0068]$. Judged TTP was generally earlier for slow-moving objects than for fast-moving ones.

\section{Discussion}

This final experiment confirmed the results found in Experiments 1 and 2, using much shorter exposure times and real stimulus objects. In this vastly different experimental environment, motion sampling was still found to have adverse effects on TTP judgments, resulting in late reactions when the object was viewed intermittently. Moreover, the response delay increased with lower sampling rates. Experiment 3 provided truly continuous motion whenever the shutter glasses were open. Thus, the sampling results cannot be attributed to artifacts of the CRT display. Time sampling continued to produce delayed TTP judgments, thus ruling out several potential extraneous factors-notably, computer-generated artifacts and cognitive extrapolation. It appears warranted to conclude that our ability to make TTP judgments is biased toward late responses as a function of sampling rate. Within limits, the effect appears to be independent of the length of stimulus ontime. These findings also support earlier proposals (Savelsbergh, 1995, p. 126; Savelsbergh et al., 1993, p. 155) that the timing of the grasping action in catching is under continuous control. This process is likewise disturbed by motion sampling. 


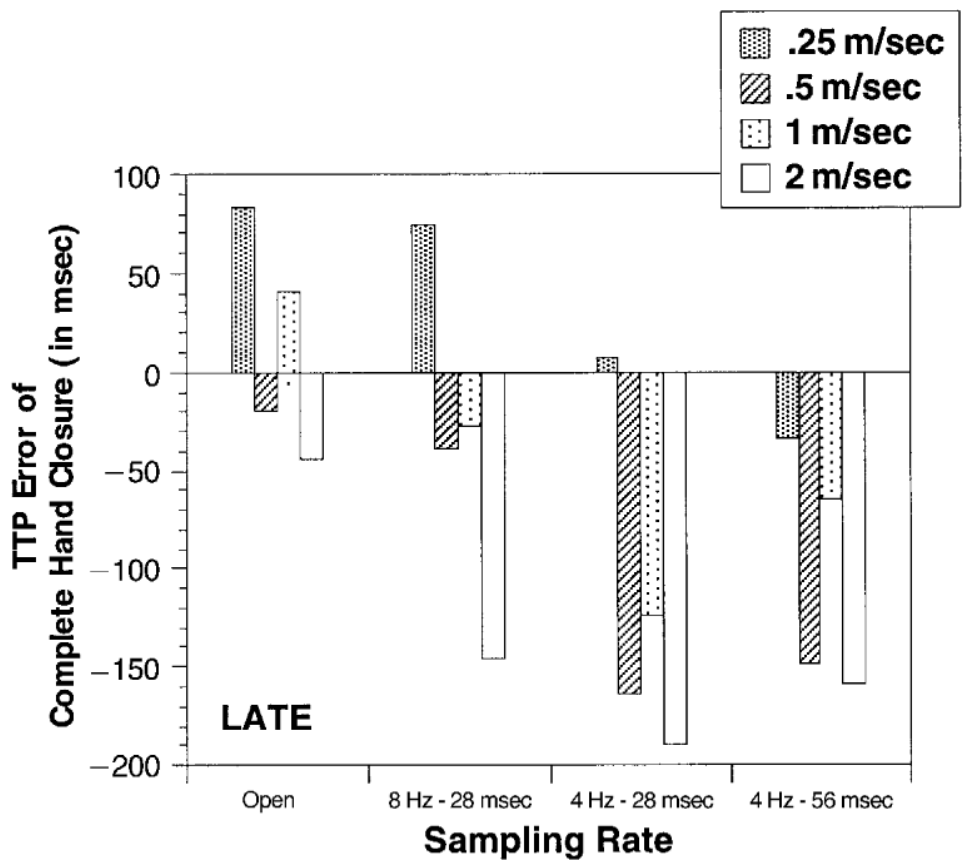

Figure 9. Average differences between maximal hand closure and true time to passage (TTP) as a function of object speed in Experiment 3. For slowmoving objects, TTP was underestimated; for fast-moving objects, TTP was comparatively overestimated.

One might wonder whether the lighting conditions in the darkened room are comparable with real-world catching situations in daylight. To ensure that no extraneous cues could be used to time the action (such as markings on the track of the BallTrAp and other reference points in the room), we kept the room lights off. However, even if performance was below daylight potentials, there is no reason to believe that room illumination would have affected some conditions preferentially. Thus, the sampling effect appears to be a robust main effect.

\section{GENERAL DISCUSSION}

\section{Sampling Effects Are Robust}

The main purpose of these experiments was to examine whether the perceptual slowing of sampled motion generalizes to arrival time judgments in depth. Using a TTC/ TTP paradigm, we replicated the frontoparallel sampling effect for sagittal motion. In comparison with continuous viewing, observers overestimated TTC/TTP, even at sampling rates as high as $18 \mathrm{~Hz}$. Both random and regular sampling intervals produced the effect. The negative effect of time sampling is not limited to simulated situations but, rather, appears to extend to natural environments. It is also robust across a variety of extrapolation times.

\section{Was Extrapolation Time a Confound?}

Could the lack of continuous perceptual support affect the accuracy of TTC judgments in a manner similar to motion extrapolation tasks done completely without perceptual support? Longer extrapolation periods between the end of the motion display and reaction time lead to velocity overestimation (e.g., Ellingstad \& Heimstra, 1969; Yakimoff, Mateeff, Ehrenstein, \& Hohnsbein, 1993). Thus, if we had found observers to overestimate sampled motion speeds, we could have reduced sampling to an extrapolation problem. And in fact, in Experiment 1, the correlation of extrapolation time and TTC errors was positive (albeit small: $r=.23, p<.0001)$. The constant stimulus durations (5 or $1 \mathrm{sec}$ ) might have posed a problem for interpreting the data: In the most extreme case that we used (i.e., $1-\mathrm{Hz}$ sampling and ontime of $14 \mathrm{msec}$ in Experiment 2), the last visible flash of the stimulus was terminated 986 msec earlier than it was in the continuous counterpart of the trial. In Experiment 3, extrapolation times were 0 for continuous stimuli and around $100 \mathrm{msec}$ for time-sampled trials. The increased extrapolation times in our sampled conditions could thus have explained earlier TTC judgments (see Yakimoff et al., 1993). However, sampling caused an effect in the opposite direction: TTC/ TTP estimates increased. Also, in the context of TTC/TTP paradigms, extrapolation times have a clear optimum of around $1 \mathrm{sec}$ (Kaiser \& Mowafy, 1993; Navarro, Banks, \& Ehrlich, 1994). Longer extrapolation times generally lead to TTC underestimation, whereas shorter extrapolation times produce the opposite effect. Typically, a crossover is found between 1 and $2 \mathrm{sec}$ of extrapolation time. For instance, Caird and Hancock (1994) found TTC overestimation for 
extrapolation times below $1 \mathrm{sec}$ and underestimation for longer extrapolation times. Thus, motion extrapolation after the visible part of the stimulus cannot account for the results.

Extrapolation time differences can explain the different baselines that we obtained between the real and the computer stimuli. The baseline shift between Experiments 1 and 2 is rather large. This might have been caused by the reduction screen's being a potentially less compelling display than the large rear projection screen used in Experiment 1 . However, we believe that the total set of stimuli constitutes a baseline with respect to which different conditions must be related in relative terms within each experiment. That is, in Experiment 1, scarce displays (all dot displays) were common, whereas Experiment 2 always displayed approaching cubes. Thus, although we find clear sampling effects within each experiment, differences between experiments need to be judged in the context of the adjacent stimuli that the observer used as a comparison standard. Trials consisting of dots led to late reactions, whereas the cube trials produced early reactions only within Experiment 1. It is known that the baseline in TTC and TTP paradigms depends on such extraneous factors as relative luminance (a factor we noticed in pilot studies). Thus, the relatively luminous cube contrasted with the lowluminance dots biased observers to perceive the target as closer and consequently produced earlier reactions (see also Castet, Lorenceau, Shiffrar, \& Bonnet, 1993). However, such a contrast was absent in Experiment 3. This does mean, of course, that our data can be interpreted only in relative terms, but not in absolute terms. We therefore do not make any strong claims with respect to the absolute TTP values.

\section{Processing Latencies Might Contribute to the Sampling Effect}

A variation of the above-mentioned kappa effect was described by Morgan $(1979,1980)$. He presented observers with an apparent motion version of a vernier acuity task. Two vertical lines (one above the other) were flashed briefly at identical azimuths as they traversed a CRT screen in apparent motion. One line, however, always flashed somewhat later than the other. It also appeared to be spatially lagging for interstimulus intervals of less than $128 \mathrm{msec}$. Morgan suggested that because the lines were perceived to be in continuous rather than discrete motion, the time sampling produced a spatial lag.

The flash-lag effect described earlier seems to be a variant of the kappa effect. It is, however, questionable whether it can indeed be explained by processing latencies between continuous and sudden-onset stimuli. This explanation put forth by Nijhawan $(1994,1997)$ has received many recent challenges. Different authors have attempted to attribute the effect to longer processing latencies of flashed stimuli (Whitney, Murakami, \& Cavanagh, 2000), to visual persistence (Krekelberg \& Lappe, 2000), to attention (for a refutation, see Khurana, Watanabe, \& Nijhawan, 2000), and to postdiction (Eagleman \& Sejnowski, 2000). Finally, Brenner and Smeets (2000) claim that the necessity to sample the continuous stimuli when comparing it with the flash is responsible for the effect.

Thus, processing latencies, which then translate into longer TTC judgments, might contribute to the effect, although the flash-lag phenomenon is phenomenally very different from sampled motion, especially when the duty cycles and sampling rates are high.

\section{Tau Theory and Sampling}

The sampling effect is not predicted by tau theory. Certainly, duty cycles of $225 \mathrm{msec}$ should suffice to extract valid TTC information. Thus, if we adhere to tau theory, we have to craft an explanation for the effect that involves modification of the tau information, such as averaging (DeLucia, 1999; Tresilian, 1993) or cognitive reprocessing (Tresilian, 1995). On the other hand, it might be more parsimonious not to do so. Evidence is accumulating that observers do not use tau information when solving TTC tasks but, rather, resort to simpler cues whenever such cues are available. For instance, Andersen, Cisneros, Atchley, and Saidpour (1999) concluded that observers use an analysis based on speed and size information, rather than tau. This is supported by effects of the size of visual obstacles, observer speed, and edge rate on judged TTC. Likewise, DeLucia and Meyer (1999) state that factors other than optical TTC information are required to model perceived collision. Such models are likely to be based on parameters that are less complex than tau, such as image velocity (Kerzel, Hecht, \& Kim, 1999), velocity combined with distance (Smeets, Brenner, Trébuchet, \& Mestre, 1996), or expansion rate (Flach, Smith, \& Stanard, in press). This is also supported by studies with drivers whose data is explained by simpler parameters (Cavallo, Laya, \& Laurent, 1986). Thus, given the questionable status of tau, adding factors to it that explain sampling is neither elegant nor parsimonious. It is certainly clear that tau theory by itself does not predict the sampling effect, but neither do the justmentioned alternate concepts.

\section{Energy Summation}

A simple energy summation model might explain the TTC estimates just as well as do more complex hypotheses. If we no longer assume that the sampling has produced a shift backward in space, TTC judgments might be based on the summed energy (e.g., luminance) that emanates from the stimulus. This is akin to the notion of a pooling mechanism, as suggested by Helson and Bevan (1967), but energy summation drops the assumption that the system prefers even spatial and temporal distributions of the samples. Pooling would predict irregular sampling to produce larger errors than does regular sampling, which is not what we found. Moreover, pooling is suggestive of a perceptual mislocalization of the target that puts the target behind its current position and can consequently only predict smaller errors than those found in our data (Experiment 2).

A simple energy summation mechanism that aggregates energy as a function of the cumulative exposure time, on the other hand, predicts the sampling effect, as well as the 
absence of a difference between random and regular sampling. It is consistent with Experiment 1, where dots were always judged to have longer TTCs than did cube objects. And the slower the sampling rate, the longer TTC was judged to be. Depending on the stimulus composition, the baseline accuracy lies somewhere in between and may shift to shorter TTCs as more big continuous objects are shown. Hence, stimulus energy appears to predict TTC judgments in a direct fashion. By all accounts (brightness, screen area, visual angle, etc.), the cube possesses more energy than does the single-pixel dot. As the stimulus is sampled, it loses some if its energy, and indeed, the fewer the samples that are provided, the slower the object appears to move. Thus, a simple-minded model of energy summation can explain our results to some extent. The energy argument receives further support from the fact that the moving starfield reduced errors only in the time-sampled cases. The spatial support that was provided by surrounding stimuli moving in unison with the target decreased the TTC errors induced by sampling of the target. If the visual system were capable of extrapolating between samples, the regular sampling at a constant frequency should have improved TTC judgments. The fact that it did not favors a simple process of energy accumulation.

Energy summation reaches its limits in Experiment 2, where reducing the ontime of the stimulus from four frames to one did not produce a change in TTC error. Thus, the model would have to be supplemented with a quantization aspect, so that energy increases make a difference only above a certain threshold. In fact, the system might utilize a critical change in energy to trigger the action of catching. Neither does energy summation explain why the type of motion sampling matters. Artificial sampling, such as that produced by strobe lighting or that produced in our experiments, appears to produce more error than does natural sampling that results from eye blinks or from objects moving behind occluders (Hecht \& Hoffman, 2000). In conditions of natural sampling, the visual system has additional information that can be used to predict when a sample will occur (by means of an efference copy of eye muscle innervation or a representation of the occluder).

Taken together, a parsimonious, albeit imperfect, lowlevel explanation of the sampling effect could consist in a quantized energy summation.

\section{Higher Level Explanations}

Parsimony does not, of course, rule out higher level explanations. For instance, representational momentum(Freyd \& Finke, 1984) might be disturbed by sampling. Just as passive observers show lesser accuracy in extrapolating movements (Larish \& Andersen, 1995), sampling might make the task harder. Also, because our representations are continuous and highly anticipatory, motion sequences that are interrupted or terminated are nonetheless thought to be represented as continuous. The theory can explain the majority of findings that extrapolation leads to slower judged speeds (e.g., DeLucia \& Liddell, 1998), but Castet's (1995) results are not predicted by representa- tional momentum. However, such higher level processes should be less pronounced when reaction times are too short to leave time for such processing. Tresilian (1995) has attempted to reconcile findings by proposing two separate mechanisms for short-range and long-range TTC estimation. Only the latter allows for cognitive processing that can influence TTC judgments. For technical reasons, it is very difficult to create complex laboratory simulations of TTP scenarios for the brief sequences (100$200 \mathrm{msec}$ ) typical for batting or catching situations in ball sports. The temporal extension of simulated events, however, poses a potential for cognitive processes to enter arrival time judgments, which may interfere with the TTP estimation process described by Lee and his colleagues (Lee, 1976; Lee \& Reddish, 1981). Quick reactions associated with preparation times of 100- $200 \mathrm{msec}$ (e.g., hitting a baseball) can be performed with remarkable accuracy; timing errors are within $\sim 30$ msec. Judgments associated with longer preparation times (or even with extrapolation of visual events beyond several seconds) usually produce more variable estimates.

We found clear sampling effects in the computersimulated trials with long extrapolation times, as well as in trials with real objects and very short extrapolation times (i.e., down to about $100 \mathrm{msec}$ ). Thus, the nature of cognitive contributions to the sampling effect is not straightforward.

\section{Background Effects}

Positive effects of the visual support lent by a moving starfield (Experiment 2) were found for sampling rates below $8 \mathrm{~Hz}$. A remaining question is the nature of the information that is provided by the starfield. Is it distance, distance change, or the observer's motion vector that becomes better specified by the supporting starfield? And why is the starfield support only facilitatory when motion is sampled?

Studies that have explicitly investigated the effect of background information on TTC judgments are inconclusive. Some of them showed no effects of the particular backgrounds used (DeLucia \& Meyer, 1999; McLeod \& Ross, 1983; Schiff \& Detwiler, 1979), whereas others found strong effects (e.g., Gray \& Regan, 2000). For instance, in the classic study by Schiff and Detwiler (1979), a filmed animated approach of a black form on a screen was used to examine effects of different backgrounds on TTC judgments. The observers were required to indicate when they thought the form (small or large at near and far distances) would reach them had it continued on the same trajectory. The form was not visible for the final $2 \mathrm{sec}$ of the approach. Background information (i.e., different types of grids) was introduced in order to provide enhanced distance, or distance change, information. None of the background manipulations was found to influence the accuracy of TTC judgments. However, the background always remained stationary while the form was moving.

A moving background, on the other hand, has been shown to produce systematic errors (Smeets et al., 1996) 
when placed in conflict with tau information. In our Experiment 2, the starfield was consistent with TTC information and seemed to have provided valuable redundant motion information, rather than lower order distance information. This suggests that local tau information was not exclusively used to judge TTC; rather, in the sampled trials, global information was used as well.

The use of global flow information in the presence of local tau specifying TTP, however, appears to be limited to sampled viewing. This result is consistent with findings by Berthelon and Mestre (1993), who investigated a quasicontinuous flow-field related to driving. They presented computer-generated scenes from the perspective of a driver on a stretch of road that curved to the left. Observers had to judge whether a car approaching on a crossroad from the right would cross before or after the driver reached the intersection. Despite the realistic animations (which contained changes in optical size, shading, and texture), the observers did not rely on global flow-field information to judge arrival times. Instead, they appeared to base their decisions on a reference cue that was given by a road sign near the intersection.

In summary, the background effect that we found only for sampled stimuli is not akin to other background effects. It is nicely explained by the energy hypothesis above. The background adds perceptual support and energy to the display and therefore compensates to some extent for the sampling effect. Continuous displays have reached a ceiling and are thus not facilitated any further. Of course, this post hoc explanation is highly speculative.

\section{Implications for Perception and Action}

The results of our experiments are consistent with Hoque's (1990) and Stewart et al.'s (1993) findings that factors other than the optical variable of tau influence TTC judgments (see also Hill, 1980). Moreover, our results might explain certain accidents and locomotion errors. For example, Kitamura, Matsunaga, and Nagao (1994) report that three quarters of cars' rear-ending stationary vehicles occur at night. They also report the seemingly counterintuitive fact that stationary vehicles were passed at closer margins (i.e., were more likely to be hit) when their hazard lights were turned on. Eight experimental observers were instructed to drive laps on an oval test track, whose edges and center line were hard to see owing to rainy nighttime conditions. On the shoulder of a straight stretch of the track, a second car was parked. A camera recorded the position of the moving car and the passing margin. Such errors could be caused by a misperception of TTC or TTP owing to time sampling, since intermittent flashing causes arrival time to be overestimated. This overestimation falsely suggests that collision is not imminent, even though it is.

Our finding that sampling produced delayed reactions in visual simulation tasks, as well as in the mimed catching situation with real objects, helps to explain Elliot et al.'s (1994) results. They found that at sampling rates of less than $10 \mathrm{~Hz}$, tennis-ball-catching performance deteri- orated considerably. Shutter glasses were used to produce motion sampling of a real scene. Longer duty cycles helped very little. These catching errors in intermittent lighting could very well be caused by TTC errors.

Our results also shed some light on the issue of continuous versus intermittent or preprogrammed control of motor action. According to the latter view, movements are preprogrammed, and TTC information is needed only a certain time before contact in order to initiate the movement. In case of continuous control, however, modulations of the movement execution still take place as a result of a constant update of the information. Evidence for continuous control in catching was found by Savelsbergh et al. (1993). In Experiment 3 of the present study, sampling affected only the final part of the catch. If TTC information serves only to initiate the movement, there should not be such an effect. Hence, it is likely that the action was continuously updated and that sampling had negative effects on the unfolding of the catch.

In summary, we favor a simple low-level explanation of the increased arrival time judgments that are associated with sampled motions. Higher level cognitive explanations are improbable-in part, because predictability of the sampling had no effect on performance. The notion that quantized stimulus energy determines TTC judgments explains both the sampling effect, as well as background effects, and the baseline shifts toward the average energy of the whole stimulus set, which we found between the experiments. Further research is needed to subject this post hoc hypothesis to direct testing.

\section{REFERENCES}

Andersen, G. J., Cisneros, J., Atchley, P., \& Saidpour, A. (1999). Speed, size, and edge-rate information for the detection of collision events. Journal of Experimental Psychology: Human Perception \& Performance, 25, 256-269.

Berthelon, C., \& Mestre, D. (1993). Curvilinear approach to an intersection and visual detection of collision. Human Factors, 35, 521-534.

BRADDICK, O. (1974). A short-range process in apparent motion. Vision Research, 14, 519-527.

Brenner, E., \& Smeets, J. B. J. (2000). Motion extrapolation is not responsible for the flash-lag effect. Vision Research, 40, 1645-1648.

CAIRd, J. K., \& Hancock, P. A. (1994). The perception of arrival time for different oncoming vehicles at an intersection. Ecological Psychology, 6, 83-109.

CASTET, E. (1995). Apparent speed of sampled motion. Vision Research, 35, $1375-1384$.

Castet, E., Lorenceau, J., Shiffrar, M., \& Bonnet, C. (1993). Perceived speed of moving lines depends on orientation, length, speed, and luminance. Vision Research, 33, 1921-1936.

Cavallo, V., Laya, O., \& Laurent, M. (1986). The estimation of timeto-collision as a function of visual stimulation. In A. G. Gale, I. D. Brown, C. M. Haslegrave, I. Moorhead, \& S. Taylor (Eds.), Vision in vehicles I (pp. 179-183). Amsterdam: North-Holland.

DAnnemiller, J. L., Heidenreich, S. M., \& BABler, T. (1997). Spatial sampling of motion: Seeing an object moving behind a picket fence. Journal of Experimental Psychology: Human Perception \& Performance, 23, 1323-1342.

DeLucia, P. R. (1999). Size-arrival effects: The potential roles of conflicts between monocular and binocular time-to-contact information, and of computer aliasing. Perception \& Psychophysics, 61, 1168-1177.

DeLucia, P. R. \& Liddell, G. W. (1998). Cognitive motion extrapolation and cognitive clocking in prediction-motion tasks. Journal of Ex- 
perimental Psychology: Human Perception \& Performance, 24, 901 914.

DeLucia, P. R., \& Meyer, L. E. (1999). Judgments about the time to contact between two objects during simulated self-motion. Journal of Experimental Psychology: Human Perception \& Performance, 25, 18131833.

DeLucia, P. R., \& Warren, R. (1994). Pictorial and motion-based depth information during active control of self motion: Size-arrival effects on collision avoidance. Journal of Experimental Psychology: Human Perception \& Performance, 20, 783-798.

Dosher, B. A., Landy, M. S., \& SPerling, G. (1989). Ratings of kinetic depth in multidot displays. Journal of Experimental Psychology: Human Perception \& Performance, 15, 816-825.

Eagleman, D. M., \& SeJnowski, T. J. (2000). Motion integration and postdiction in visual awareness. Science, 287, 2036-2038.

Ellingstad, V. S., \& Heimstra, N. W. (1969). Velocity-time estimation as a function of target speed and concealment extent. Human Factors, 11, 305-312.

Elliot, D., Zuberec, S., \& Milgram, P. (1994). The effects of periodic visual occlusion on ball catching. Journal of Motor Behavior, 26, 113 122.

Flach, J. M., Smith, M. R. H., \& Stanard, T. (in press). Perception and control of collisions: An alternative to the tau hypothesis. In M. McBeath (Ed.), Navigational principles used by humans, animals, and machines. London: Sage.

Freyd, J. J., \& FinKe, R. A. (1984). Representational momentum. Journal of Experimental Psychology: Learning, Memory, \& Cognition, 10, 126-132.

GELDARD, F. A. (1975). Sensory saltation: Metastability in the perceptual world. Hillsdale, NJ: Erlbaum.

GotTSDANKER, R. M. (1952). The accuracy of prediction motion. Journal of Experimental Psychology, 43, 26-36.

Gray, R, \& Regan, D. (1999). Motion in depth: Adequate and inadequate simulation. Perception \& Psychophysics, 61, 236-245.

GRAY, R, \& REGAN, D. (2000). Simulated self-motion alters perceived time to collision. Current Biology, 10, 587-590.

Hecht, H., \& Hoffman, D. D. (2000). Extrapolating visual motion: Effects of spatial and temporal sampling. Manuscript submitted for publication.

Hecht, H., Oesker, M., Kaiser, A. Civelek, H., \& Stecker, T. (1999). A perception experiment with time-critical graphics animation on the World-Wide Web. Behavior Research Methods, Instruments, \& Computers, 31, 439-445.

Hecht, H., van Doorn, A., \& Koenderink, J. J. (1999). Compression of visual space in natural scenes and in their photographic counterparts. Perception \& Psychophysics, 61, 1269-1286.

Helson, H. (1925). The psychology of Gestalt. American Journal of Psychology, 36, 494-526.

Helson, H., \& Bevan, W. (1967). Contemporary approaches to psychology. Princeton, NJ: Van Nostrand.

Helson, H., \& King, S. M. (1931). The tau effect: An example of psychological relativity. Journal of Experimental Psychology, 14, 202217.

HILl, B. L. (1980). Vision, visibility, and perception in driving. Perception, 9, 183-216.

Hoque, M. M. (1990). An analysis of fatal bicycle accidents in Victoria (Australia) with a special reference to nighttime accidents. Accident Analysis \& Prevention, 22, 1-11.

KaISER, M. K., \& Mowafy, L. (1993). Optical specification of time-topassage: Observers' sensitivity to global tau. Journal of Experimental Psychology: Human Perception \& Performance, 19, 1028-1040.

Kerzel, D., Hecht, H., \& Kim, N. G. (1999). Image velocity, not tau, explains arrival-time judgments from global optical flow. Journal of Experimental Psychology: Human Perception \& Performance, 25, 1540-1555.

Khurana, B., Watanabe, K., \& Nijhawan, R. (2000). The role of attention in motion extrapolation: Are moving objects "corrected" or flashed objects attentionally delayed? Perception, 29, 675-692.

Kitamura, F., Matsunaga, K., \& Nagao, I. (1994). Influence of looking at hazard lights on car-driving performance. Perceptual \& Motor Skills, 78, 1059-1065.
Krekelberg,B., \& Lappe, M. (2000). A model of the perceived relative positions of moving objects based upon a slow averaging process. $\mathrm{Vi}$ sion Research, 40, 201-215.

Larish, J. F., \& ANDERSEN, G. J. (1995). Active control in interrupted dynamic spatial orientation: The detection of orientation change. Perception \& Psychophysics, 57, 533-545.

LEE, D. N. (1976). A theory of visual control of braking based on information about time-to-collision. Perception, 5, 437-459.

LEE, D. N., \& RedDISH, P. E. (1981). Plummeting gannets: A paradigm of ecological optics. Nature, 293, 293-294.

Lee, D. N., Young, D. S., Reddish, P. E., Lough, S., \& Clayton, T. (1983). Visual timing in hitting an accelerating ball. Quarterly Journal of Experimental Psychology, 35A, 333-346.

Loomis, J. M., Da Silva, J. A, Philbeck, J. W., \& Fukusima, S. S. (1996). Visual perception of location and distance. Current Directions in Psychological Science, 5, 72-77.

Macknik, S. L., Fisher, B. D., \& Bridgeman, B. (1991). Flicker distorts visual space constancy. Vision Research, 31, 2057-2064.

McLeod, R. W., \& Ross, H. E. (1983). Optic-flow and cognitive factors in time-to-collision estimates. Perception, 12, 417-423.

Michaels, C. F. (2000). Information, perception, and action: What should ecological psychologists learn from Milner and Goodale (1995)? Ecological Psychology, 12, 241-258.

Miller, R. J., \& Patterson, R. (1995). Influence of flicker on perceived size and depth. Perception \& Psychophysics, 57, 604-613.

Morgan, M. J. (1979). Perception of continuity in stroboscopic motion: A temporal frequency analysis. Vision Research, 19, 491-500.

Morgan, M. J. (1980). Spatiotemporal filtering and the interpolation effect in apparent motion. Perception, 9, 161-174.

Navarro, R, Banks, M. S., \& Ehrlich, S. (1994).Local and global methods for estimating time-to-contact and time-to-passage. Investigative Ophthalmology \& Visual Science, 35, 1999.

NIJHAWAN, R. (1994). Motion extrapolation in catching. Nature, 370, $256-$ 257.

NiJHAWAN, R. (1997). Visual decomposition of colour through motion extrapolation. Nature, 386, 66-69.

Ono, A. (1976). A study of the literature on the interrelations between subjective time, distance, and speed. Tohoku Psychologica Folia (Tomus XXXV, Fasc. 1-4). Tohoku University, Sendai, Japan.

Regan, D., \& Vincent, A. (1995). Visual processing of looming and time to contact throughout the visual field. Vision Research, 35, 1845 1857.

Rogowitz, B. (1984). The breakdown of size constancy under stroboscopic illumination. In L. Spillmann \& B. R. Wooten (Eds.), Sensory experience, adaptation, and perception (pp. 201-213). Hillsdale, NJ: Erlbaum.

Savelsbergh, G. J. P. (1995). Catching "Grasping tau": Comments on J. R. Tresilian (1994). Human Movement Science, 14, 125-127.

Savelsbergh, G. J. P., Whiting, H. T. A., \& Bootsma, R. J . (1991). "Grasping" tau. Journal of Experimental Psychology: Human Perception \& Performance, 19, 315-322.

Savelsbergh, G. J. P., Whiting, H. T. A., Pijpers, J. R., \& van Santvoord, A. M. M. (1993). The visual guidance of catching. Experimental Brain Research, 93, 146-156.

SCHIFF, W., \& DeTWILER, M. L. (1979). Information used in judging impending collision. Perception, 8, 647-658.

SCHIFF, W., \& OlDAK, R. (1990). Accuracy of judging time to arrival: Effects of modality, trajectory, and gender. Journal of Experimental Psychology: Human Perception \& Performance, 16, 303-316.

Sidaway, B., Fairweather, M., Sekiya, H. \& McNitt-Gray, J. (1996). Time-to-collision estimation in a simulated driving task. Human Factors, 38, 101-113.

Smeets, J. B. J., Brenner, E., Trébuchet, S., \& Mestre, D. R. (1996). Is judging time-to-contact based on "tau"? Perception, 25, 583-590.

Stewart, D., Cudworth, C. J., \& Lishman, J. R. (1993). Misperception of time-to-collision by drivers in pedestrian accidents. Perception, 22, 1227-1244.

Stewart, D., Cudworth, C. J., \& Lishman, J. R. (1997). Response to the critical note 'Correcting some misperceptions of time-to-collision,' by James R. Tresilian. Perception, 26, 237-241.

ToDD, J. T. (1985). Perception of structure from motion: Is projective 
correspondence of moving elements a necessary condition? Journal of Experimental Psychology: Human Perception \& Performance, 11, 689-710.

Tresilian, J. R. (1993). Four questions of time to contact: A critical examination of research on interceptive timing. Perception, 22, 653-680.

Tresilian, J. R. (1994). Approximate information sources and perceptual variables in interceptive timing. Journal of Experimental Psychology: Human Perception \& Performance, 20, 154-173.

Tresilian, J. R. (1995). Perceptual and cognitive processes in time-tocontact estimation: Analysis of prediction-motion and relative judgment tasks. Perception \& Psychophysics, 57, 231-245.

Tresilian, J. R. (1997). Correcting some misperceptions of time-tocollision: A critical note. Perception, 26, 229-236.

van der Kamp, J., Savelsbergh, G. J. P., \& Smeets, J. B. (1997). Multiple information sources in interceptive timing. Human Movement Sciences, 16, 787-821.

WANN, J. P. (1996). Anticipating arrival: Is the tau margin a specious the- ory? Journal of Experimental Psychology: Human Perception \& Performance, 22, 1031-1048.

Wertheimer, M. (1912). Experimentelle Studien über das Sehen von Bewegung [Experimental studies on motion perception]. Zeitschrift für Psychologie, 61, 161-265.

Whitney, D., Murakami, I., \& CaVAnagh, P. (2000). Illusory spatial offset of a flash relative to a moving stimulus is caused by differential latencies for moving and flashed stimuli. Vision Research, 40, 137-149.

Wong, E., \& Weisstein, N. (1984). Flicker induces depth: Spatial and temporal factors in the perceptual segregation of flickering and nonflickering regions in depth. Perception \& Psychophysics, 35, 229-236.

Yakimoff, N., MateefF, S., Ehrenstein, W. H., \& Hohnsbein, J. (1993). Motion extrapolation performance: A linear model approach. Human Factors, 35, 501-510.

(Manuscript received October 25, 1999; revision accepted for publication August 9, 2001.) 